\title{
Modeling of heat consumption in a greenhouse using experimental data
}

\author{
Stawomir Grabarczyk ${ }^{1, *}$ \\ ${ }^{1}$ Institute of Civil Engineering, Faculty of Civil Engineering, Mechanics and Petrochemistry, Warsaw \\ University of Technology, Łukasiewicza 17, 09-400 Płock, Poland
}

\begin{abstract}
The use of experimental research results to teach artificial neural networks was aimed at determining the relationship between heat consumption and measured variables. The mechanism of changes in heat consumption was determined by changes in external climate parameters, microclimate conditions in the greenhouse and parameters describing the functioning of the technical equipment of the facility. The accuracy of modeling the heat consumption in the case of changes in the properties of the building's external partitions has been determined. In a greenhouse, this is related to the functioning of an additional movable curtain - a thermal screen. MLP networks - multilayer perceptrons with hidden layers proved to be particularly useful for predicting changes in heat consumption. In the analysis attention was paid to the accuracy of modeling depending on the size of the measurement data set. The sources of information are collections with different intervals between registered measurement records, which were collected during the tests carried out during the full calendar year.
\end{abstract}

\section{Introduction}

Modeling of heat consumption in a greenhouse is desirable in order to properly shape microclimatic conditions for cultivation. This is also important from the point of view of determining the actual thermal needs in such facilities depending on the requirements imposed during the year. It is possible to predict heat consumption in order to reduce it, based on current outdoor climate measurements and using this information to control greenhouse heating systems in real time [1]. With the historical outdoor climate data sets, one can determine the operating costs of the facility for different locations.

Thermal climatic factors have a significant influence on heat consumption in the greenhouse. Modern greenhouses are often equipped with movable shading and energysaving screens, which significantly limit the influence of solar radiation in the plant growing zone. Additional curtains also limit heat losses in a greenhouse [2]. Movable thermal screens in a greenhouse have a significant impact on heat supply in this type of buildings. Artificial neural networks exhibit the greatest effectiveness primarily in the modeling of complex non-linear processes with variable characteristics, which cannot be clearly described mathematically [3-4]. Several studies were made on the greenhouses to

*Corresponding author: slawomir.grabarczyk@pw.edu.pl 
predict greenhouse climatic parameters [5-6]. Proper representativeness of source data should contain at least one year of measurements [7-8].

The aim of the analysis was to determine the suitability of artificial neural networks to forecast heat consumption and to determine the effects of ANN applicability in a greenhouse, a building with a significant impact of changing external conditions.

\section{Greenhouse description}

The basis of the performed analysis are measurements of greenhouse microclimate parameters, heat energy consumption and external climatic conditions. The mono-span greenhouse has an active curtain installed inside - a thermal screen, reducing heat loss in the period of low temperatures and allowing shading of the greenhouse growing surface during the high values of solar radiation intensity. The sidewalls of the greenhouse are thermally insulated by sealing with the air bubble foil. The greenhouse is located in a garden farm and is intended for year-round cultivation in pots. Basic information about the greenhouse is presented in the Table 1.

Table 1. Characteristics of the greenhouse.

\begin{tabular}{|c|c|c|}
\hline \multirow{3}{*}{$\begin{array}{c}\text { Mono-span } \\
\text { greenhouse }\end{array}$} & Location & Skierniewice (Poland) \\
\cline { 2 - 3 } & Floor area & $1250 \mathrm{~m}^{2}$ \\
\cline { 2 - 3 } & Volume without thermal screen & $6187.5 \mathrm{~m}^{3}$ \\
\cline { 2 - 3 } & Volume with thermal screen & $5471.6 \mathrm{~m}^{3}$ \\
\cline { 2 - 3 } & $\begin{array}{c}\text { Global heat transfer coefficient } \\
\text { without thermal screen }\end{array}$ & $6.68 \mathrm{~W} /\left(\mathrm{m}^{2} \cdot \mathrm{K}\right)$ \\
\cline { 2 - 3 } & $\begin{array}{c}\text { Global heat transfer coefficient } \\
\text { with thermal screen }\end{array}$ & $4.20 \mathrm{~W} /\left(\mathrm{m}^{2} \cdot \mathrm{K}\right)$ \\
\hline $\begin{array}{c}\text { Thermal screen } \\
\text { ULS 16 }\end{array}$ & $\begin{array}{c}\text { Energy saving } \\
\text { shading }\end{array}$ & $60 \%$ \\
$65 \%$
\end{tabular}

\section{Materials and method}

Calculations of the predicted heat consumption in the greenhouse were made using the Statistica Neural Networks software. Data sets constituting the basis for teaching artificial neural networks contained a number of variables registered with an interval of 1 hour and 10 minutes, which corresponds to the number of records, respectively 8,760 and 52,560 for the full year of registration. Teaching of the artificial neural network was used with the cause-and-effect method, i.e. indirect method, and the most important input variables that could affect the heat consumption in the greenhouse were taken into account. To this end, in the first stage of teaching the network, those variables whose significance was irrelevant were eliminated. The basis for selecting the variables was the sensitivity analysis. The set of variables in the first stage of analysis is presented in Table 2.

Heat consumption was qualified both as the output variable and as the input variable with lag. The lag values accepted in the data set with the 1 hour interval included observations up to 6 hours back, while in the set with the interval of 10 minutes, up to 12 hours back of observations. This allowed obtaining more accurate calculation results and taking into account in the modeling of heat consumption in its previous values. Heat consumption in a greenhouse is affected by the variability resulting from the fluctuations of external climate conditions, changing preset microclimate parameters and functional technical equipment, as well as from the implementation of the heating system control process. Due to significant fluctuations in the observations of the heat meter, it was decided 
to perform parallel modeling of heat consumption with values smoothed with the use of a signal filter.

Table 2. The set of variables in the first stage of analysis.

\begin{tabular}{|c|c|c|}
\hline Symbol & Variables & Unit \\
\hline$T_{i}$ & air temperature & ${ }^{\circ} \mathrm{C}$ \\
\hline$\varphi_{i}$ & internal air humidity & $\%$ \\
\hline$T_{e}$ & external air temperature & ${ }^{\circ} \mathrm{C}$ \\
\hline$\varphi_{e}$ & external air humidity & $\%$ \\
\hline$w$ & wind speed & $\mathrm{m} / \mathrm{s}$ \\
\hline$I$ & total solar radiation & $\mathrm{W} / \mathrm{m}^{2}$ \\
\hline$\psi$ & light intensity & $\mathrm{klx}$ \\
\hline$T S P$ & thermal screen position & $\%$ \\
\hline$V P$ & vents position & ${ }^{\circ}$ \\
\hline$T_{g-10, T_{g-50}}$ & ground temperature $-10 \mathrm{~cm},-50 \mathrm{~cm}$ & ${ }^{\circ} \mathrm{C}$ \\
\hline$Q_{m}$ & actual heat consumption & GJ \\
\hline$Q_{m-l a g}$ & lag heat consumption & GJ \\
\hline
\end{tabular}

The signal filter used several times performs smoothing with the use of mean and mobile median and creates a series while maintaining the characteristic properties of a series of primary data [9]. As a result of using filter $4235 \mathrm{H}$ the following transformations are performed: a 4 points moving median centered by a moving median of 2 a 5 point moving median, a 3 point moving median, and a 3-point weighted moving average using Hanning weights $(0.25,0.5,0.25)$. Residuals are computed by subtracting the transformed series from the original series, steps 1 through 4 are then repeated for the residuals and the transformed residuals are added to the transformed series. In practice, this filtering method often produces a smooth series while maintaining the salient characteristics of the original series.

Artificial neural networks were tested in the calculations: multilayer perceptrons with one hidden layer (MLP) and networks with radial basis functions (RBF). The teaching process was carried out in MLP networks by back propagation of error, scaled conjugated gradients and Quasi-Newton (Broyden-Fletcher-Goldfarb-Shanno) training algorithm, while for RBF networks by K-means (determination of radial neurons weight) and K-nearest neighbors (radius-deviation determination) and pseudo-inverse algorithm.

In order to reduce complexity in networks with radial RBF base functions, the number of hidden neurons was limited to 128 , and in the case of multilayer perceptrons, their number was limited to 15 . In the case of neural networks MLP both linear, hyperbolic tangent, exponential and logistic functions of neuron activation were used. So in the training phase, a larger part of the database $(70 \%)$ was used to train the network and the remaining part of the database was used in the validation (15\%) and testing phase $(15 \%)$.

\section{Result and discussion}

The review of the results indicated a significant unsatisfactory adaptation possibility of RBF type networks and positive effects of energy consumption forecasting obtained by MLP networks. The results of teaching of artificial neural networks MLP on measured data sets are presented in Table 3. 
Table 3. The results of heat consumption prediction for the best a neural networks - measured data.

\begin{tabular}{|c|c|c|c|}
\hline \multirow{2}{*}{ Result } & \multicolumn{2}{c|}{ Set } \\
\hline Parameter & Subset & $8760(1$ hour $)$ & $52560(10$ min. $)$ \\
\hline \multirow{3}{*}{ Performance } & Training & 0.844 & 0.719 \\
\cline { 2 - 4 } & Validation & 0.841 & 0.716 \\
\cline { 2 - 4 } & Test & 0.831 & 0.705 \\
\hline \multirow{3}{*}{ Error } & Training & 0.0090 & 0.0012 \\
\cline { 2 - 4 } & Validation & 0.0086 & 0.0011 \\
\cline { 2 - 4 } & Test & 0.0092 & 0.0012 \\
\hline \multirow{2}{*}{ Quotient Of } & Training & 0.535 & 0.718 \\
\cline { 2 - 4 } & Validation & 0.541 & 0.730 \\
\cline { 2 - 4 } & Test & 0.558 & 0.729 \\
\hline Neural Network & Mlp & $8-9-1$ & $6-8-1$ \\
\hline Training Algorithm & Bfgs & 79 & 174 \\
\hline \multirow{2}{*}{ Activations } & Input & tanh & logistic \\
\cline { 2 - 4 } & Output & exponential & logistic \\
\hline
\end{tabular}

Among the tested networks, the ones that gave the best results in the modeling of heat consumption were presented. The basis for the assessment and selection of the best network was the criterion related to the minimization of the error of the validation set, i.e. the set allowing independent checking of the quality of the network during its teaching.

The sensitivity analysis of variables affecting the obtained results of modeled heat consumption is presented for the best networks in Table 4.

Table 4. The sensitivity analysis of variables affecting the obtained results of modeled heat consumption.

\begin{tabular}{|c|c|c|c|c|}
\hline \multirow{3}{*}{ Variables } & MLP 8-9-1 & MLP 6-8-1 & MLP 8-9-1 & MLP 6-15-1 \\
\hline & 8760 & 52560 & 8760 & 52560 \\
\hline & \multicolumn{2}{|c|}{ Measured data } & \multicolumn{2}{|c|}{ Measured and filtered data } \\
\hline$T_{i}$ & 1.53 & 1.28 & 1.03 & 1.21 \\
\hline$T_{e}$ & 2.63 & 1.52 & 1.10 & 2.16 \\
\hline$I$ & 1.34 & 1.36 & 1.16 & 1.17 \\
\hline$T S P$ & 1.21 & 1.02 & 1.01 & 1.09 \\
\hline$Q_{m-1}$ & 1.85 & 3.76 & 3.48 & 108.36 \\
\hline$Q_{m-2}$ & 1.19 & 1.40 & 8.28 & 20.16 \\
\hline$Q_{m-3}$ & 1.15 & - & 7.28 & - \\
\hline$Q_{m-4}$ & 1.39 & - & 5.16 & - \\
\hline
\end{tabular}

The significance of the previous observations of heat consumption on the output variable result in both raw and filtered data sets is characteristic. For example, in raw data sets with an interval of 10 minutes, variables carrying information about two previous indications of heat consumption are very important in the proper determination of the output variable. In the data set with an interval of 1 hour, the four previous heat consumption indications are relevant.

Table 5 presents the results of heat consumption prediction for the best a neural networks MLP divided into teaching, validation and test sets. 
Table 5. The results of heat consumption prediction for the best a neural networks MLP - measured and filtered data.

\begin{tabular}{|c|c|c|c|}
\hline \multirow{2}{*}{ Result } & \multicolumn{2}{c|}{ Set } \\
\hline Parameter & Subset & $8760(1$ hour $)$ & $52560(10 \mathrm{~min})$. \\
\hline \multirow{3}{*}{ Performance } & Training & 0.898 & 0.986 \\
\cline { 2 - 4 } & Validation & 0.896 & 0.986 \\
\cline { 2 - 4 } & Test & 0.896 & 0.987 \\
\hline \multirow{3}{*}{ Error } & Training & 0.0061 & 0.00002 \\
\cline { 2 - 4 } & Validation & 0.0058 & 0.00002 \\
\cline { 2 - 4 } & Test & 0.0059 & 0.00002 \\
\hline \multirow{2}{*}{$\begin{array}{c}\text { Quotient of } \\
\text { deviation }\end{array}$} & Training & 0.953 & 0.168 \\
\cline { 2 - 4 } & Validation & 0.921 & 0.167 \\
\cline { 2 - 4 } & Test & 0.921 & 0.157 \\
\hline Neural network & MLP & $8-9-1$ & $6-15-1$ \\
\hline Training algorithm & BFGS & 125 & 327 \\
\hline \multirow{2}{*}{ Activations } & Input & tanh & tanh \\
\cline { 2 - 4 } & Output & linear & logistic \\
\hline
\end{tabular}

The performance of the best model to predict the heat consumption in greenhouse showed that MLP method is applicable, based on measured and filtered values in a 10 minutes data set. The assessment in this respect is determined by the measures determined on the basis of the test set, i.e. the set of which data was not used in the neural network learning process. Since the errors for the test set are close to the errors for the training set, the estimation of the value of the explanatory variable should be considered to be at a satisfactory level.

Finally in the training, validation and testing phases of ANN implementation, there is no significant difference between the measured and filtered data and the predicted data in a 10 minutes data set. Fig. 1 shows the comparison of predicted data by ANN to measured and filtered data of heat consumption.

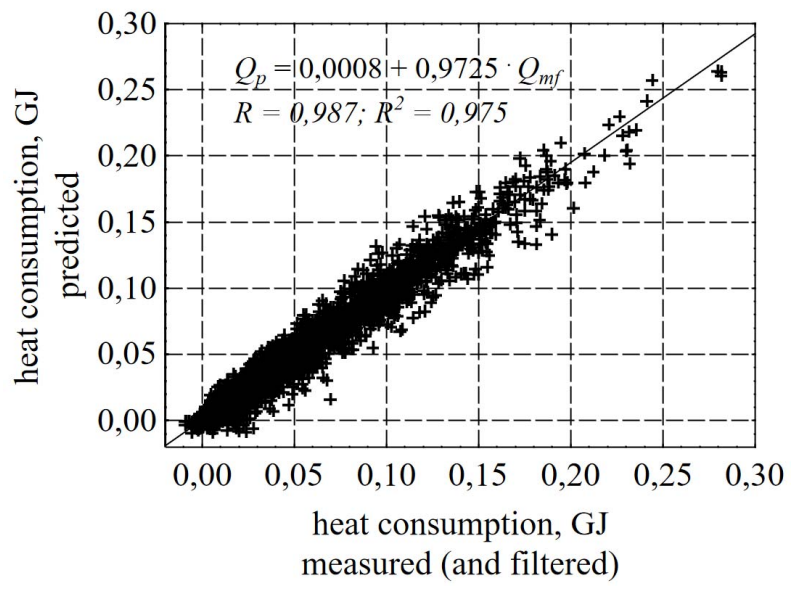

Fig. 1. Comparison between the measured and predicted values by the artificial neural networks MLP 6-15-1 model for the heat consumption - test set. 
An especially important parameter in the evaluation of artificial neural networks is the quotient of deviations, which subtraction from the unity determines the part of the explained variance. For the best neural networks for greenhouses with measured data, the quotient of deviation is 0.558 ( 1 hour) and $0.729(10 \mathrm{~min})$, while for a neural network predicting heat consumption in a greenhouse with data filtered 0.921 (1 hour) and 0.157 (10 $\mathrm{min})$. The results of analysis indicate that filtering the heat consumption signal indicated by the heat meter results in better matching quality.

\section{Conclusions}

As a result of the implementation of a series of calculations, it was found that multilayer perceptrons (MLP) with one hidden layer gave the best effects of modeling the heat consumption in a greenhouse. The indirect method of modeling heat consumption in a greenhouse has been successfully applied in the case of the tested object. The causal method can be used to determine the thermal needs of greenhouses, if historical climate data are known.

A better quality of artificial neural network matching in modeling heat consumption in 10-minute data sets is obtained by using pre-observation. The use of a signal filter to smooth the observation of heat consumption allowed better prediction of the variable, while increasing the importance of pre-observations.

The results indicate high cognitive abilities resulting from the use of artificial neural networks MLP to predict heat consumption in a greenhouse. It is expected that the use of neural networks will allow for modeling heat consumption in a greenhouse in conditions other than those observed, which was the basis for teaching the neural network.

\section{References}

1. Z.S. Chalabi, B.J. Bailey, D.J. Wilkinson, Comp. Electr. Agric. 15, 1 (1996)

2. Y. Zhang, L. Gauthier, D. de Halleux, B. Dansereau, A. Goseselin, Agric. For. Meteo. 82, 227 (1996)

3. F. Fourati, Neurocomputing 139, 138 (2014)

4. M. Taki, Y. Ajabshirchi, S.F. Ranjbar, A. Rohani, M. Matloobi, Energy and Build. 110, 317 (2016)

5. T. Boulard, S. Wang, J. Agric. Eng. Res., 75, 27 (2000)

6. P.M. Ferreira, E.A. Faria, A.E. Ruano, Neurocomputing 43, 51 (2002)

7. I. Seginer, Comp. Electr. Agric. 18, 167 (1997)

8. R. Lineker, I. Seginer, Math and Comp in Simul. 65, 19 (2004)

9. Statistica for Windows, Vol. III: Statistics II (StatSoft Inc. Tulsa, 1997) 